\title{
Choreographing the City: can dance practice inform the engineering of sustainable urban environments?
}

\begin{abstract}
In this paper, we aim to demonstrate that choreographic practice - its ways of thinking about, designing and embodying movement - has the potential to offer new insights into the engineering of sustainable urban infrastructure and environments. In order to make such an argument, we identify urban mobility as an ideal starting point to discuss the potential overlaps between engineering and choreographic thinking. In order to do so, we firstly and briefly outline the notion of sustainability as it pertains to a key area of city engineering - urban transport. Drawing on critical theory about the broad set of issues impacted by the way mobility in cities is designed and managed, we suggest that transport is one of the most critical issues in creating socially and environmentally sustainable urbanism. Critiques of engineering are drawn upon to show how its working cultures and epistemologies prevent it from undergoing the transformation that would allow it to effectively address the issues posed by sustainable transport. The focus of the paper is what we believe to be a comprehensive survey of the few conceptual and practical experiments that have been undertaken in using choreographic techniques to explain or design the way people move in cities, in scholarship and design practice. We conclude by arguing that there is great potential to expand on these experiments as ways to address the cultural and epistemological limits within engineering, and calling for practice-based research that shows what impact this would have on its processes and outcomes.
\end{abstract}

\section{Introduction}

In this paper we argue that the artistic practice of dance could offer engineering new ways of describing, modelling, and designing movement, whilst it faces great challenges in finding solutions to the need for healthy, sustainable cities. We start by outlining why urban mobility - as a product of both cultural and infrastructural factors - is a global environmental issue, and why engineering is limited by its own epistemologies in its ability to enact the needed level of change. Starting from the hypothesis that an expert practice dealing with movement could offer urban engineering new ways of working, we attempt to offer a systematic survey that has not been available before of the ways in which techniques and concepts related to dance have been used to described, model, design, and assess urban mobility. We conclude that there is demonstrable potential in this intersection of dance and engineering that has not been fully articulated, and clear need to test the impact of this kind of knowledge-sharing through real- 
world research that could, through new forms of practice, offer different outcomes in urban mobility, and later other realms of urban engineering.

\subsection{What is engineering?}

Since the $19^{\text {th }}$ century engineers have led the way in delivering the infrastructures that have modernised cities and allowed them to support the levels of health, accessibility, and communication that most urbanites in the Global North can expect today. Engineers built railways to spur the Industrial Revolution, sewerage systems that eliminated many urban diseases, rolled out telecommunications systems and delivered large-scale public events such as Olympic Games across the world. Though engineers' interventions in cities are often at an infrastructural level, and are subject to little public debate in comparison to architecture and urban design that are much more visible (as argued by ) they are key in shaping the values produced by public spaces. For example, the engineering of urban transport systems, from train networks to streetscapes, encodes assumptions about who moves, for what purposes, and what the experience of using these systems should be, which are rarely challenged or debated within engineering practice or in the public sphere.

As advances in technology continue to accelerate in the $21^{\text {st }}$ century and as social change mirrors this pattern, engineers must adapt their practices just to keep up, and to continue to contribute to society in a meaningful and positive way. This change is driven primarily by the pressing needs of climate change adaptation and mitigation that requires a move away from carbon intensive resources and towards more responsive and adaptive systems; by the rise of big data, deep learning and artificial intelligence, which will replace the need for human designers; and by global urbanisation, which will concentrate and intensify populations' consumption needs.

\subsection{Why dance?}

With this in mind, the engineering, infrastructure and construction industries, with their large institutions and companies, often siloed approaches (Blockley and Godfrey, 2017), and somewhat dated education systems, are at risk of falling short. Without substantial and sustained attention and creative approaches to transformation the industry could become as Ramírez and Seco, (2012) describe, "victims of ingrained inertia, that will require a decisive push if they are not to become hidebound organizations, enslaved to the past, with few signs of future effectiveness and efficiency." In terms of its role in the design and construction of the built environment, engineering is at an important moment: perhaps, as Kanter (2015) implores, "we are stuck not only with aging infrastructure but also with obsolete ways of talking about 
it." If the engineering industry continues to rely on solutions based on limited notions of efficiency, the social and environmental costs will continue to be paid by urban citizens. Alternately, it can look to fundamentally change its assumptions by incorporating new realms of knowledge and practice.

Though seemingly distantly related, both choreography and engineering design are creative processes that design materials through time and space, they are both taught formally, are developed through practice and experience, have known outputs, physical and economic constraints and desired social outcomes. Both practices deal with curating form and movement to shape personal experiences of space. These practices have developed independently and have emerged from different schools, which may have different underlying assumptions and processes that we will explore through this work. Dance practice - its ways of thinking about, designing and embodying movement - has the potential to offer new insights into the design of sustainable urban environments.

\section{The Challenge for Engineering}

Urban mobility, understood as broader than urban transport and describing all the means by which and reasons for which people move in cities, is an obvious starting point for thinking about how to incorporate new forms of practice derived from dance into engineering, and the focus of nearly all of the small pool of literature that have already been made to link dance and urban design. Mobility is also a key focus for engineering in cities, including distinct fields such as infrastructure planning, vehicle design, streetscape specification and delivery, and crowd management by design - and though engineering is equally one of the core design practices that plans the way mobility is managed in cities it is not explicitly mentioned in any of this literature. In this section, we give a brief overview of the reasons for which mobility is so crucial for urban sustainability, and the role and shortcomings of engineering specifically in addressing this.

\subsection{Mobility as environmental sustainability}

Cities, among other things, are systems for movement which require the consumption of energy. The ways cities are planned to support movement, then, is clearly impactful for their energy consumption and subsequently their environmental sustainability. A recent (late $20^{\text {th }}$ and early $21^{\text {st }}$ century) turn towards mobility in the social sciences though, has brought a focus onto the way individual or group experiences of the city are fundamentally shaped by these systems of movement - whether in terms of the freedom to move around the city versus the inability to do so, or the freedom to be stable versus being forced to migrate, and so on. 
Transport systems also afford access to social associations and resources, and their distribution shapes the city culturally in doing so (Docherty et al., 2008, p. 84). As demand for mobility within cities has grown throughout the $20^{\text {th }}$ century to answer the need for "copresence for social, economic, familial, and legal connections" (Bridge and Watson, 2011, p.165) and distances have increased, engineers have sought solutions based on an implicit cultural ideal of individual freedom of movement that has often led to the prioritisation of carbased infrastructures. The resulting increase in car use has in many metropolitan areas, particularly in North America, been both cause and effect of a pattern of deconcentration and decentralization (Docherty et al., 2008, p. 85). In turn, car use has increased reliance on fossil fuels and deaths from pollution and road accidents, while decreasing access to employment and opportunities for active, low-cost modes of transport such as walking and cycling (Low and O'Connor, 2013, p. 4). A need for mobility to be sustainable has been recognised (though the basis for measuring sustainability is by no means a matter of consensus), and unfettered individual automobility is seen no longer to be viable in environmental terms (lbid., p. 6). We can see this for example in Transport for London's 'Healthy Streets' approach, which prioritises active transport in the capital.

\subsection{Cultural issues in mobility}

Environmentally, then, urban mobility is a global issue. To make sustainable the consumption of energy by urban transport there will need to be dramatic changes in infrastructural conditions, such as the availability and attractiveness of non-polluting shared transport modes. Such change will also be the result of billions of individual decisions, taken in the context of highly varying levels of choice, about where to travel and how. These decisions, taken together, could be described as a culture of mobility, consisting of norms and expectations around how people move. As it was in the proliferation of automotive infrastructure, engineering is of course deeply implicated in the development of new solutions for sustainable transport. The question remains though, as to whether it will be able to create radical new solutions using the same tools it has historically adopted, by intervening at large scale in the physical fabric of the city or by introducing new technologies, when these cultures of mobility are so powerful.

Whilst the environmental challenge of urban mobility lies in its volumes at urban, regional, and global scales, a full understanding of what drives and constrains the individual movements that accrete to form these flows is surely needed within engineering if it is to find genuinely new solutions. At the same time, mobilities theory has revealed how movement in cities is a cultural practice rather than simply a function of necessity. As well as being the predominant mode within which the city is experienced, movement is shaped by and constitutive of socio- 
economic inequalities. 'Urban travel is not just about getting from point A to point B', argues Ole Jensen. 'It is about producing and re-producing the city and the self in a complex relationship involving mobility cultures and different types of mobility knowledge' (Jensen, 2006, 161). Acarón has highlighted the extremely divergent experiences of differently gendered bodies in movement spaces, for example, impacted upon by the way those spaces are designed (Acarón, 2016). In recent years, physical and mental health have become key motivators in attempts to encourage active modes of transport that combine exercise with mobility and consume less energy. Encouraging these choices at an individual level has been shown to require a combination of changes to both physical context (the built environment) and cultural context (shared beliefs and attitudes) (Lanzendorf and Busch-Geertsema, 2015). Transport for London's recent adoption of a strategy for 'Healthy Streets' identifies qualitative goals for street design: they should feel 'relaxed', act as 'welcoming places for everyone to walk, spend time in and engage in community life', and encourage the choice of walking, cycling, and public transport (Transport for London, 2017). Achieving these goals will require engineers to translate between diverse definitions of, and ways of evaluating, qualitative notions such as 'welcoming' and 'relaxed', and develop responses from a wider knowledge base than is currently possible within the practice, as we go on to argue here.

\subsection{The limits of engineering practice in transforming cultures of mobility}

In his anthropology of engineering practice, Louis Bucciarelli points to an empiricist worldview focused 'primarily on the individual posing and resolving of questions of a scientific/instrumental nature', which he critiques as 'necessary but not sufficient if design is to succeed' (Bucciarelli, 2002, 219-220). Engineering is also specifically a positivist science, heavily reliant on assumptions of predictable causal relationships between physical phenomena (Douglas et al., 2010). These critiques are particularly pertinent where the object of engineering is urban transport, that forms a set of infrastructural conditions for the sociocultural complexity of everyday urban life. As suggested above, transportation in cities has myriad implications beyond the quantified imperatives it is usually predicated upon (desired or existing volumes of transport flow, economic growth goals and funding constraints). With its positivist approach to framing problems to be addressed, however, engineering limits the degree to which it can incorporate the complexity and conflict within these implications. Lam's (2017) critique of the London Borough of Hackney's cycling infrastructure interventions, for example, demonstrates the ways in which the reductionist engineering approach to design failed to account for the social and political nature of cycling adoption. Particularly, Lam argues, the rationale adopted that all users of the infrastructure could be characterised as having the same needs led to the design of an infrastructure that fails to accommodate the racial, gendered and classed nature of cycling. 
Reduction is necessary to solve any problem with empirical data, but as cities are characterised by a particularly dense overlaying of modes of information, they present unusual challenges to this reduction. Measurable qualities dealt with in engineering are overlaid with and produce unquantifiable characteristics that vary subjectively and often work through metaphorical language in everyday or 'lay' descriptions of place - places and infrastructures that are warm or cold, feel safe or threatening for example, affecting the degree to which people are willing to use them. Such language is absent from engineering practice from brief to assessment. Without an ability to describe the objects of engineering in these terms and employ qualitative empirical modes to understand what creates these effects, engineering has no hope of affecting sustainable changes in mobility culture.

Engineering devises systems to meet desired needs set by customers (who in a transport context are often governmental bodies) that are framed epistemologically in a way suited to the information engineers can work with. Kroes et al. explain that "in a process of translation, specification and reduction, these needs are transformed into functional requirements and these again into design specifications. The list of design specifications - usually a list of physical parameters - may be taken to be the stated objective of an engineering-design project. If the designed object meets all the specifications, it is supposed to be able to perform the desired function" (Kroes et al., 2009, 566). The epistemological structures of problemframing in engineering, then, pre-define the possible range of solutions that can be modelled and the specifications that can be tested in those models. If, as Douglas et al. (2010) have called for, there was allowance in engineering for embodied, interpretive, subjective viewpoints as a valid knowledge-generators, might the assumptions about the specifications that engineering can design to also be expanded? The aim in broadening engineering's epistemological horizons in this way is not only that it can deal with the hard-to-quantify qualities of urban mobility, but that it finds new ways to do design, that widen the range of possible solutions it can envisage.

Kroes et al. explain how this rational/creative distinction in engineering constrains techniques as well as solutions: "not only the processes by which new options are added to the engineers' agenda..., but also the processes leading up to new ways of realizing existing artefacts.... [It] is precisely in those situations that decisions have to be made about what, given the assessments of all the prevailing constraints and uncertainties, may be possible to achieve and worthwhile to pursue... It is in the search for new technological possibilities that creativity and rationality appear to go hand in hand" (Kroes et al., 2009, 537). In other words, rather than jumping to end scenarios, as is the case in some more utopian projects to re-imagine what engineering can do, ${ }^{i}$ creativity must be better applied in the everyday processes of 
gathering information and using it to model potential outcomes, in order to add 'new options to the agenda'. In doing so, assumptions around what is 'possible' and 'worthwhile' for engineers to do may change. So, epistemological limitations in engineering operate at several interrelated stages in its design process: the way it describes the world and frames problems to be solved; a range of solutions constrained by the framing of the problem; and the designing of those solutions with the same tools and datasets as the ones that created the problem. Drawing on external value systems, from the arts for example, to evaluate its own products could reveal to engineering new problems to be solved in new ways.

Of course, calls for a better integration of humanist modes of knowledge into engineering are not new. Grimson et al. have called for a richer historical and philosophical context to engineering education, arguing that "in the future engineers will have to face up to a long-term convergence between technological and liberal arts education", without which "engineers would not be in a position to provide any type or level of leadership required in our more complex society" (Grimson et al., 2008, 20). Though such aims do not seem far-fetched, they face an industry whose culture allows that the UK's Royal Academy of Engineering can state in a report on engineering education that 'universities must continue to teach 'core engineering' and not dilute course content with peripheral subject matter' (Spinks et al., 2006, 16).

\subsection{The potential for new approaches}

As it stands then, urban engineering is constrained by a relatively stable position in which it has a rigid epistemological structure that enables it to manage highly complex projects and focus its future imaginaries on hypothetical technological scenarios, rather than change in its everyday cultures and crafts. To conceptualise the barrier to change this represents it is useful to draw from Holling's (1973) ecological systems theory. We can say that the current system of engineering practice has reached a 'steady state' where the processes, actors and tools are largely known and, although dynamic and changeable, are relatively stable. The stable states that have emerged in engineering practice are centred on nineteenth and twentieth century assumptions. Engineers build large scale infrastructure in fundamentally the same way as 40 years ago, because it appears to work within the rational knowledge framework engineering has created for itself. The failure to challenge the worldviews engineering itself is based upon threatens to dig us deeper into systems that are unsustainable environmentally as much as they are in terms of their socio-cultural and psychosomatic effects on the lives of urbanites. Holling would argue, however, this is not the only stable state that could have emerged and that there are other potential system configurations with differing levels of effectiveness. Our hypothesis is that by better understanding the way other forms of expert 
practice, and particularly artistic ones, frame problems, generate knowledge, and test solutions, both the fundamental assumptions and the means of doing engineering design could be updated.

\section{Choreographing Urban Mobility}

Hanoch-Roe has suggested that "the fields of architecture and landscape architecture could benefit from looking into related arts such as music and dance that inherently have to deal with temporal and movement aspects, to derive a new and more complete understanding of the process of conveying these elements into the design of space" Hanoch-Roe $(2007,86)$. Other calls have been made for collaborative work between dancers and urbanists to explore these parallels further (see McCormack, 2008). A small number of scholars, as will be seen below, have used language or concepts derived from choreographic practice as descriptive tools for urban mobility. Even more rarely, choreographic techniques have been applied to design and modelling processes within urban mobility. However, there has been no systematic study or taxonomy of this research, and engineering itself, with its distinct empirical modes of practice, has been entirely absent from such calls, in favour of the hybrid arts of architecture and landscape design. Though an exhaustive survey of such work is beyond the scope of a single paper, we hope to build on the sparse but promising set of ideas at this intersection to suggest that there is clear potential for the development of a field of scholarship and practice, that requires further theoretical research and practical experimentation going beyond analogical uses of dance as a concept for describing mobility and testing instead the application of its epistemological and creative tools to engineering practice itself.

\subsection{Description}

\subsubsection{Dance in the city}

A discussion of the relationship between urban space and dance is not new. Many dance scholars have reflected on the way their own movement practices are acted upon and act back upon the built environment. Schiller and Rubidge, speaking about the incorporation of urban place and form into dance practices taking place beyond traditional performance spaces, argues that through experiments in and with sites, architectures have been reframed by dance as movement systems. They describe "choreographic situations", improvised movements in the urban environment, as ways of revealing the topology of urban landscape to be kinaesthetic rather than static, and revealing places as sets of actions rather than of objects (Schiller and Rubidge, 2014, p. 53). Victoria Hunter, amongst others, has mobilised choreographic thinking as a form of analysis of the built environment, extending George Perec's method of 'exhausting place' through a catalogue of objects and events by using the 'body-self' to list elements from a kinaesthetic perspective (Hunter, 2017). Such accounts do 
not attempt to deal with the way cities are designed, taking them instead as complete objects that can be encountered through different epistemologies derived from dance practice. However, these modes of observation and knowledge-production through improvised movement and thinking with the body - which we are describing as epistemologies for describing the context within which design is to be done - are clearly beyond and therefore potentially enriching to the current state of data collection within engineering.

Research from dance practice also raises some important points about terminology, which will be helpful throughout. Dance, as we are using it, is an art form consisting of multiple styles, usually understood from a non-dancer perspective as a kind of art work to be experienced but also from a professional perspective including a whole set of practices that lead to the creation of that work. One of those practices, and the key aspect of dance that we are interested by, is choreographic practice, which is the specific process by which the set of movements within a dance work is conceived, modelled, tested, communicated from choreographer to dancer, rehearsed, and so on. Choreographic techniques, then, are ones used within choreographic practice to do that conception (i.e. using improvisation within a set of constraints or trying to embody concepts and feelings in movement), communication (i.e. using notation or demonstration) and so on. Choreographic thinking, as we understand it, is the application of these kinds of techniques to other realms than dance, as in Victoria Hunter's use of bodily thinking to provide a 'Perecquian' taxonomy of kinaesthetic elements in an urban environment.

\subsubsection{Place ballet}

Within the broad realm of urban studies, dance as an art form has used as a way to evoke a social coherence, even a collective art work, in the way patterns of movement emerge from the negotiation between bodies and concrete form in urban settings. Following Jane Jacob's reference to the 'sidewalk ballet' (Jacobs, 1987 [1961]) the geographer David Seamon uses ballet as a central metaphor to build an account of urban place around patterns of three fundamental components of urban mobility - movement, rest, and encounter (Seamon, 1979) - rather than as a static arrangement of space. The configuration of streets and other routes across a neighbourhood - its static morphological character - shape the "time-space routines" of its inhabitants: individual day-to-day trajectories followed through this network of routes as spatial configuration becomes learned in an embodied and unconscious manner analogous to a dancer's bodily incorporation of a pattern of choreographed pathways across a stage. This, as Seamon acknowledges, is essentially another language to describe what has been observed in detail in the sociological theory of urban morphology known as space syntax (Hillier, 2004) a statistical analysis system using quantitative data to model the outcomes of urban plans in terms of mobility and land-use value, that could arguably be framed as an 
engineering approach to urban design. Seamon (2007) explicitly develops the notion of place ballet as a way to enrich space syntax's focus on quantities of movement with an understanding of its qualities. He argues for the necessity of including the three-dimensional qualities of bodily movement and gesture in a model of urban movement, whereas space syntax and other modes of transport engineering tend to focus on two dimensional patterns of trajectories. These "body-routines", as Seamon describes them, are habitual, learned physical responses to the immediate material or functional qualities of spaces such as the height of a kerb, a bump in the road, or the raising of an arm at the bus stop. So, ballet, as a form of dance art, is used as a metaphor to bridge between the qualitative aspects of bodily gesture and the measurable configuration of routes through the built environment. In an ethnographic study of Sydney's commuter journeys, Symes (2013) adapts the notion of place ballet to an observational framework that allows for an enriched understanding of how individual bodies experience and respond to urban form. He observes that commuting is a "virtuosic form of travel, involving an array of corporeal accomplishments and embodiment" (Symes, 2013, p. 556) as well as audio cues, collective improvisation and movement phrases. However, though this works as a poetic metaphor for a movement-based model of urban place, it does not go further than the metaphor to suggest in more detail how the practice of ballet itself, with the system of language and notation used to design it, might inform the engineering of the streetscapes themselves.

\subsubsection{Scoring the traffic intersection}

David Prytherch offers a step in this direction in his analysis of urban road intersections as a "high-stakes ballet of urban life" (2015, p. 45). Prytherch also seeks to argue that "a fully relational view of mobilities starts with our haptic sense of touch and kinaesthetic sense of bodily motion" in relation to several materials: "space (choros) and time (chronos), but also more embodied conceptions of space as topos (concrete place/landscape) and time as kairos (embedded/event time)" (Ibid., p. 48). His wider conceptual framework is somewhat like Seamon's synthesis of time-space and bodily routines, then, but Prytherch offers more detailed parallels between the ways choreography and transport engineering organise time and space to shape patterns of movement.

Focusing on traffic intersections, where trajectories of large numbers of bodies and vehicles cross one another in a constrained space and create the potential for significant danger, Prytherch draws attention to several ways that circulation is structured choreographically. Movement through intersections, he argues, is guided by a pre-determined system encoding a set of assumptions about the desired qualities of that movement. Firstly, the physical design of intersections constrains movement to varying degrees: cars cannot drive through wall or 
barriers, and do not generally mount kerbs, so these solid elements create a stage that delimits the boundaries of possible movement. The shape of this stage is dependent on way different users are taken into account, which itself is an ideological decision: Prytherch's research in Hamilton, Ohio, revealed design guidelines that prioritise the need for vehicles to pass through with the minimum interference, reflecting the dominance of automotive over pedestrian culture. Within the ultimate physical boundaries of the stage, "choreography in real time depends on "control", through the imposition of a score. Analogously, in the traffic intersection, a system of markings and signals - stop lines, markings between traffic lanes, and red lights - all suggest limits to vehicular movement without physically constraining it, while a green man is an invitation for the pedestrian to commence the specific action of crossing the street, like a phrase in a dance. Making clear that this score is suggestive and not deterministic, Prytherch highlights the constant improvisation required by users encountering the many different configurations of bodies that are possible within the spatial framework suggested by the markings.

Improvisation also suggests the possibility for transgression against or beyond the score (a freedom not explicitly recognised by Seamon) on behalf of users, using the relationship between a choreographic score and improvisation by performers within that to analogise the potentially unpredictable relationship between engineering design and the actions of individuals within the framework it tries to impose. These laws and norms are not made visible within the space itself, but carried within subjects as a disciplined physical response to a set of stimuli. Prytherch therefore likens them to a system or school of choreography that disciplines dancers in specific ways, the ignorance of which comes with the threat of recriminations whether on a legal or aesthetic basis respectively. Via this useful framework, in which bodily response, material design, road markings, and legislation are all interwoven. This system of analysis based in dance, he argues, could 'manifest quite different orderings, which could produce more justice among the diverse users of the street, not all of whom are (or should be) in cars'.

\subsubsection{Embodied space}

Shrinking in scale again, from Prytherch's dance-based account of transport intersections, choreographer Thania Acarón (2016) draws on concepts from dance theory to draw attention to the way different kinds of bodies act and are acted upon when mobile in urban space. The space of the body has long been seen, as Acarón describes, as a region of three-dimensional space that extends beyond the physical boundary of the skin. Edward Hall's oft-cited theory of 'proxemics' identifies an extremely specific, measurable set of concentric radii around the body that are characterised by varying kinds of interaction: from intimate, to personal, social, 
and public space (see figure 1). Bourdieu's and Lefebvre's notions of social space are similar, suggesting that a set of invisible relationships define a definite region of sociability extending from and centred around the body. Acarón, though, argues that these ignore the importance of "intrapersonal dimensions" (Acarón, 2016, p. 140) and advocates for the application of knowledge from "body-based art forms" approaches to better integrate concepts of space into social theory. Choreographer Rudolph Laban's notion of kinesphere, for example: a spherical space defined by the extent of the limbs that has been highly influential in the design of movement in theatre and choreography but largely lacking from analysis of social space.

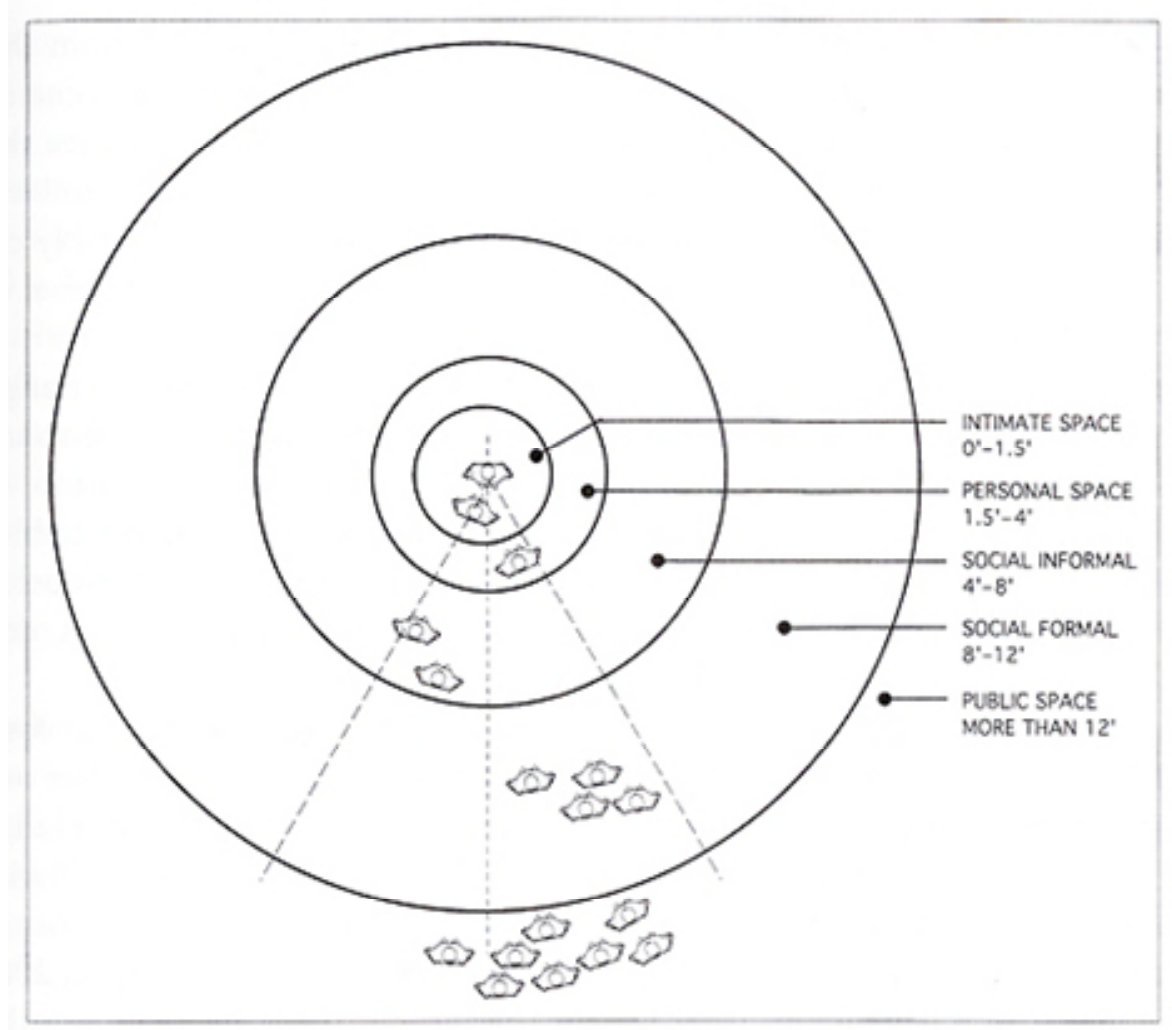

Figure 1: Diagram of Edward Hall's theory of proxemics

Borrowing and extending upon Setha Low's term embodied space, Acarón synthesises these ideas, that all agree on the centrality of the body, and adds emphasis upon "the body's ability to effect and receive change through movement" (Acarón, 2016, p.141). In doing so, she proposes a helpful set of spatial categories for the way embodied space is constituted and interacts with built and social environments: portability, extensibility, social flexibility, and transversality (Ibid., p. 142). Rather than the fixed, symmetrical bubble of personal space or the kinesphere, embodied space is an uneven zone usually weighted towards the front, following the visual organs, but sometimes reshaped or developed in other directions through physical training such as that undertaken by the dancer. The ability to access or roam through 
different spaces, as described by the "portability" of embodied space, is always a factor of both the body's level of ability or disability and the degree to which control over its agency is enacted, through spatial design that restricts or excludes for example. Embodied space is also often 'extended' through inhabitation or possession of other objects. Acarón gives the example of the driver in a car: "There is interplay between the body executing movements inside the car and reactions to the environment occurring externally. The extensibility of the space may even elicit bodily reactions, such as, for example, gasping when the car hits another car's bumper slightly while parking or an increased heart rate with acceleration beyond the speed limit" (p. 143).

So, where Prytherch reads the traffic intersection as an interplay of people and vehicles, Acarón's framework blurs the line between the two, allowing drivers and pedestrians each to be described as the loci of moving, embodied spaces in which the former (that of the driver) has greater portability and extension than the latter. Acarón refers to a boundary dance of "constant extensions and contractions" at the meeting of different embodied spaces, whether they be extended or not by technologies such as vehicles. The performing of gender in public, she argues, often involves spatial behaviours such as minimizing or maximising embodied space. Even where actual physical contact does not occur, men (usually, as suggested by Acarón) often assert privileged use of space by forcing minimisation on the embodied space of women in what are described as the "daily boundary dances of gender domination" (Acarón, 2016, p. 144). This framework goes beyond analogical comparisons to dance by applying dance theory to analysis of the size and shape of embodied spaces in relation to objects of engineering such as transport vehicles.

\subsection{Modelling and design}

Dance, as we have seen, has occasionally been employed as a descriptive tool in scholarship relating to urban mobility. Much more rarely, tools from choreography have been incorporated into the practice of urban transport design in the form of modelling and design tools. As recounted by Peter Merriman, the landscape architect Lawrence Halprin approached streets, roads and freeways as active spaces to be carefully designed, 'scored' and choreographed to produce particular movements, experiences emotions and affects for motorists, pedestrians and local communities" (Merriman, 2011). Together with his wife, the choreographer Anna Halprin, Lawrence Halprin developed two approaches addressing this lack by incorporating choreographic practice (the tools of dance making) in different ways into his own urban design projects: a workshop method for participation in urban planning; and a notation system for movement through the city. As above, dissecting the epistemological and practical 
assumptions of these approaches demonstrates the potential value, and pitfalls, in different modes of exchange between engineering and dance.

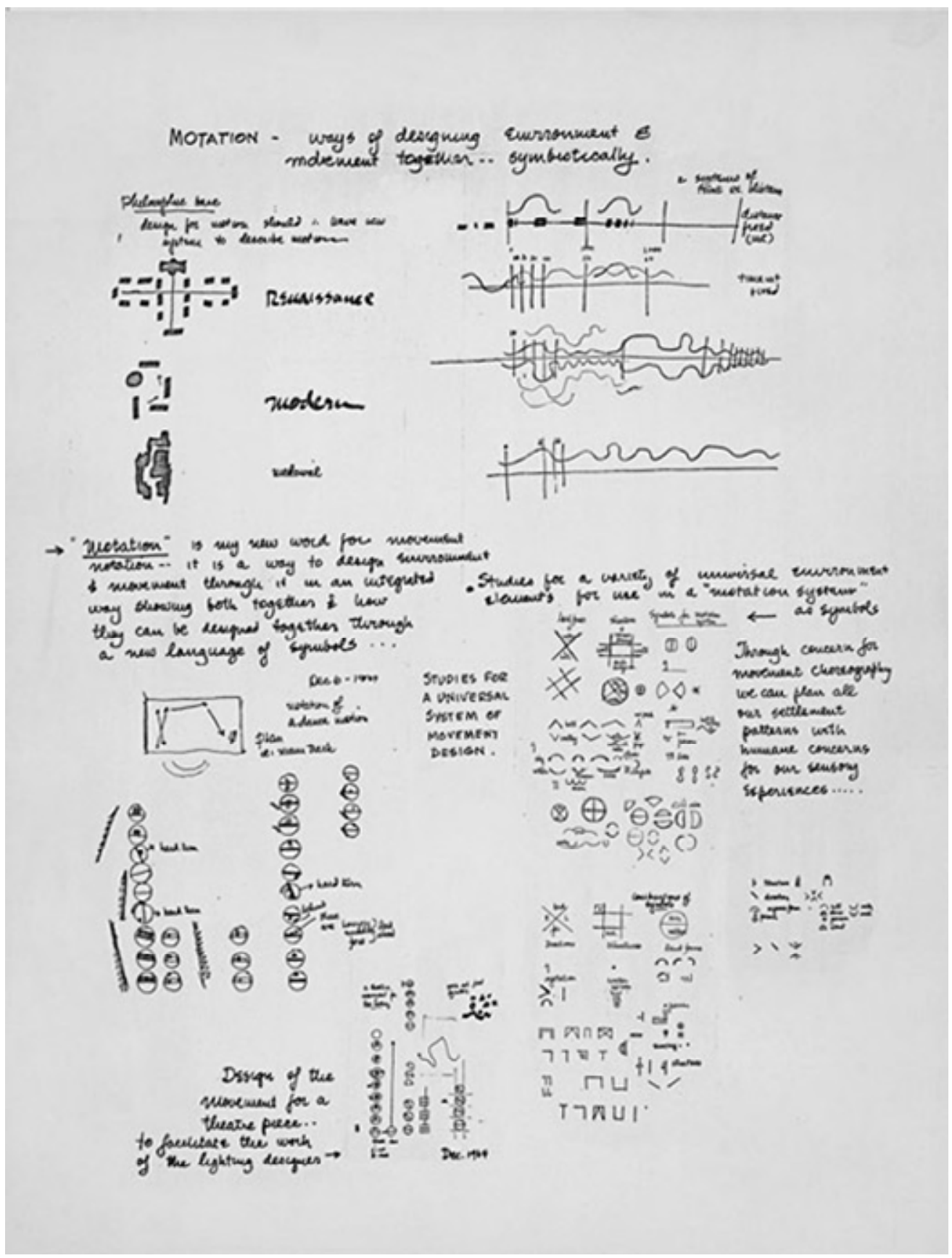

Figure 2: Lawrence Halprin Motation notes (Source: http://dataisnature.com/)

Halprin understood city as "an environment for choreography" which "must be experienced through movement" (Halprin 1963, 193, quoted in Merriman, 2011, 102). He argued, though, that because architects were taught to design in plans, elevations and models that are inherently representational - rather than describing practices, activities or movements - they are limited in their ability to explicitly design for the latter. Instead, Halprin wanted to develop 
a form of notation that would allow the designer to use movement as a starting point to generate form as opposed to form dictating movement (Hirsch, 2014). Halprin $(1963,208)$ cited in Merriman (2011) explains "[designers or planners] cannot design the movement as he has no tools to do so." Music and dance - art forms that are produced through a set of bodily movements in relation to particular technologies and spaces - are commonly guided by notation systems specifying those movements in different ways. Inspired by this, Halprin developed a system of movement notation - or "motation" - to aid the 'symbiotic' design of both environments and trajectories through those environments. Motation consisted of a series of 26 symbols for particular elements that can be encountered in a journey, alongside a linear representation of the journey itself and in relation to a time scale. This system could act as a descriptive tool to recall specific characteristics of a journey, of the city from a moving viewpoint, as opposed to the traditional architectural of structural drawing that supposes a static point of view. Equally, it was intended to act as a design tool aiding the conception of a route in relation to its three-dimensional environment, or what we might call a score for a choreography of moving relationships between bodies and architectures.

Merriman's account of Halprin's work deems motation a significant innovation in design process, challenging the design's focus on static spaces with a mobile perspective. Nonetheless, Merriman also highlights the limitations of this approach, that perhaps explain why it did not develop into an established design tool: "his published notations focus largely on the visualities generated by particular embodied movements and actions... with little sense of the multi-sensory, kinaesthetic dimensions of performing mobile embodied actions" (Merriman, 2011, 105). Referring back to Seamon's descriptive framework, we might say that Halprin developed a method for designing time-space routines in relation to the built environment (where Seamon stopped at offering terms to describe them) but failed to the impact of sensorial and material qualities of space on body-routines and gestures, or indeed the fine-grain choreographic awareness of the three-dimensional unevenness of, and conflicts between, embodied spaces.

Urbanist Kevin Lynch, along with Appleyard \& Meyer, also developed notation system for highways, noting that when perceived in movement "the kinaesthetic sensations are like those of the dance" (Appleyard et al., 4). They developed a notation system recording qualities of experience along a trajectory through urban infrastructure - near vs distant attention, moving vs static feature, texture, colour, and width of road, tempo of attention and so on - proposing that designers could use this system to plan journeys with coherent and stimulating rhythmic patterns as opposed to seeing infrastructural design from the static perspective of the traditional plan. Galia Hanoch-Roe, though, in her survey of scoring in architectural design 
that recounts both Lynch et al. and Halprin's experiments, argues "it is evident that these diverse attempts to date have not offered a comprehensive, systematic way of scoring the spatial linear experience" (Hanoch-Roe, 2007, p. 111).
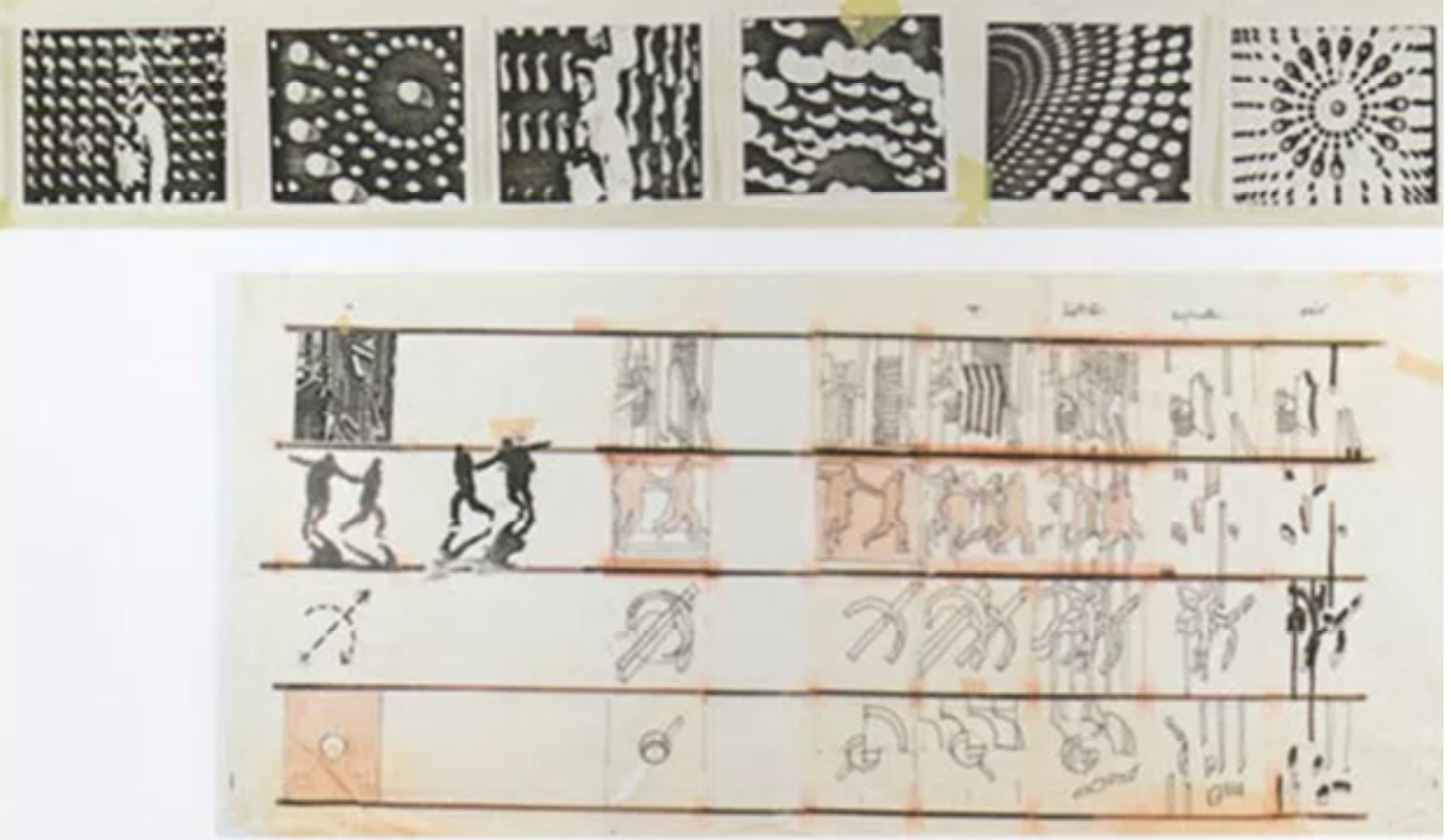

Figure 3: Bernard Tschumi's Screenplays and The Manhattan Transcripts (Source: Kato and Glynn, 2016, 8)

\subsection{Embodied knowledge}

Notation in choreography has also always faced its own methodological challenges in representing the three-dimensional complexity of trajectory, gesture, and rhythm of which dance movement is constituted, and its attempts to do so are what means it is of such potential value to the engineering of urban mobility, which otherwise models the movement of human bodies through a spatial system in much more reduced terms. As Kato and Glynn have argued, "analogue and digital notational systems for documenting choreographic movement provide promising alternative strategies for spatial design" (Kato and Glynn, 2016, 6). Like Merriman and Hanoch-Roe they note failings in attempts to incorporate these strategies into architecture, but also the limitations of choreographic notation itself: "the dynamics of interaction between user and space fail to be captured" (Ibid., 6). Attempts to transcribe movement into a frozen two-dimensional form, as they allude, will inevitably lose information in the process - information that would be essential for the incorporation of such techniques into engineering. 

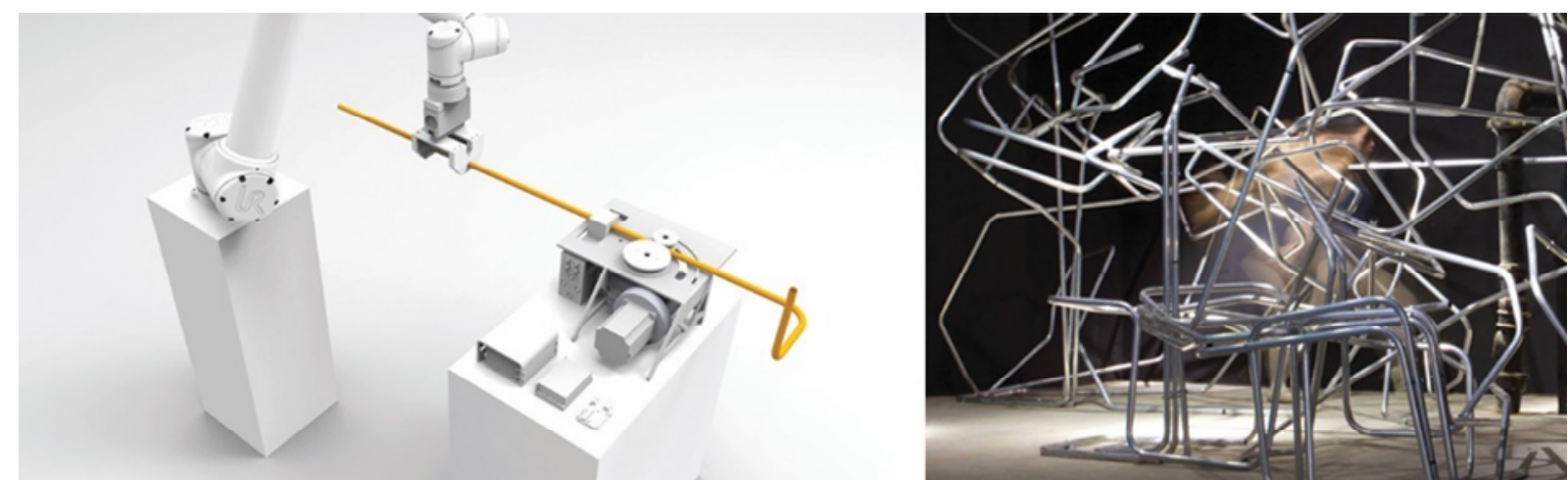

Figure 4: "UR 10 Robot and bespoke end effectors / Fabricating performance" (Kato \& Glynn, 2016, 12)

In response, Kato and Glynn demonstrate how to create a circular feedback process between movements and interactive three-dimensional models through a prototype system in which dancers were tracked using a motion capture system. This system guided a robotic fabrication system bending aluminium tubing into a three-dimensional structure tracing a rationalised representation of the accumulated gestures within the space, shaped by a set of rules and the constraints of the material. The result is "an opportunity to express a notational system spatially" and "to sense and touch the intent of a performance that emerges within the complexity of movement" (Kato \& Glynn, 2016, 12), offering a near-lossless set of spatial information about aggregated movement pathways at full scale. On this basis, they argue, design decisions could be made in a feedback loop between rational thought, embodied attention, process, and re-imagining. This kind of full-scale three-dimensional information is non-discursive, in that it cannot be fully reconstituted in description, and to some degree nonrepresentational in that is a real aggregation of material traces of movement as opposed to a set of symbols intended to stand in for those movements as in notation. In this sense, Kato and Glynn's system evokes the practice of 'marking' within dance - the carrying out of a set of actions that simplify a dance so as to allow the body to become accustomed to its spatial and temporal (or rhythmic) patterns. David Kirsh, based on experiments with both designers and choreographers to understand the role of the body in thinking processes, argues that marking is much more powerful cognitively than a mental simulation of an outcome in being able to understand its effects (Kirsh, 2011). The failures in the social and environmental effectiveness of design solutions highlighted by Bucciarelli occur where people involved in different parts of the engineering process, each with its own language and "object-world", attempt to interface with one another using visual and linguistic metaphors to represent complex processes to engineers in other realms (Bucciarelli, 2002, 224). Where elements of a larger system must interface with one another, a "vulgar" language is found but with the indeterminacy inherent when translation takes place across domains that may not have literally interchangeable concepts. Non-discursive full-scale modelling combining the 
embodied knowledge of gestures with technologies for three-dimensional notation, as a way of avoiding representation in symbols that can be misinterpreted (as described variously by Kato \& Glynn and Kirsh) seem to offer potential as a technique within these translational moments in engineering.

\section{Conclusion: systematising and testing choreographic thinking in urban mobility}

Though linked by their desire to incorporate ideas from dance into the design and analysis of urban mobility, the propositions referred to in this paper are spread across at least 50 years and emerge from very different realms of scholarship and practice. As far as we can tell, these propositions have not been systematically analysed and evaluated, nor have they been applied specifically to engineering practice, with its key role in designing socially and environmentally sustainable urban mobility. We have attempted here to survey these propositions and to conclude will offer the beginnings of a theoretical framework for the relationship between dance and engineering practice. This kind of framework, we hope, will be of value in laying the groundwork for further theoretical development as well as practical experiments whose aims would be to test the impact of these news ways of thinking and working on innovation in engineering, and consequently its ability to improve urban sustainability via much-needed changes in cultures of mobility.

The modes with which dance practice has been applied to urban mobility can generally be grouped as either object-oriented or process-oriented. Object-oriented thinking uses ideas from dance as a way to describe the existing built environment and forms of movement through it. Description of the built environment within engineering consists of collecting data about the context within which new solutions will be delivered, and is also involved in the assessment of the effect of those solutions. Description of the world always has some kind of epistemology that determines what kind of observations can be made, the format of data they lead to, and so on. Seamon and Jacobs, by invoking ballet as a metaphor for describing movement patterns through a neighbourhood street network, draw attention to data that is not traditionally included within the engineering epistemology: aesthetic qualities of relationships between individual trajectories, gestures that accompany trajectories, and the way those gestures are patterned by concrete objects within the environment for example. Acarón goes beyond this metaphorical invocation of dance as an art form to say that the embodied interactions between different users of the built environment is literally a dance - a movementbased negotiation between different embodied spaces - and thus that theories developed within dance scholarship for analysing the differential ways bodies form a space around them can be used to analyse that dance. Her framework offers the potential to engineering of describing spaces not only in terms of volumes of users, but how much space those users 
inhabit according to gender and socio-cultural inequalities. Prytherch takes an analogical approach to using the art form of dance to describe traffic intersections, not only saying that movement is 'like' a ballet but that these intersections have constitutive parts that are analogous to those within a dance work: a stage, a score, a system of movement, and interpretation. The relationship between the intended score for movement - as expressed by traffic signals, lines and so on - and the way users actually behave - transgressions against those intentions - might be understood better by engineering as interpretation of an intended system of movement if it was framed as improvisation.

Prytherch suggests this kind of description - via the analogy of a dance work - could help improve urban design processes, but does not say how exactly it would be incorporated. The experiments that can be described as process-oriented however, have focused more on the techniques of doing design for urban mobility than on description of the world as-it-is. Lawrence Halprin's movement workshops with members of the public used group work methods developed within the dance practice of Anna Halprin, his wife, as a form of participation in design. This represents an attempt to address an issue similar to that noted by Bucciarelli, of knowledge becoming reduced as it is passed between realms (in this case lay and expert) by using movement as a form of collaboration to conceive, test, and communicate designs with their potential users. His and Kevin Lynch's experiments in notating the qualities of trajectories through mobility infrastructures such as highways were developed through description of existing forms, but intended as design tools for planning experience from the mobile perspective of their users, as notation is used in choreography to plan out patterns of dance movement. These scores are representational of movement in forms that incorporate more qualitative variables than those of engineering. David Kirsh has shown that more complex ideas can be conceived and dealt with by the brain if physical is used to externalise thought processes, in what is called embodied thinking. Kato and Glynn suggest a technological system for making movement visible three-dimensionally in real time as a way to iterate and re-iterate its design. These latter examples are non-representational in that they use movement as a thinking tool rather than as a way of encoding an end result in representational form.

So, we argue, various elements of dance practice have been demonstrated as having potential for introducing new ways of working into the engineering of urban mobility: applying dancebased concepts as metaphors, analogies, or sets of analytical tools for describing and assessing the objects of engineering (relationships between the built environment and cultures of mobility); or applying choreographic techniques such as embodied thinking, notation, improvisation and movement-based collaboration to the design and modelling processes 
within engineering. This is by no means intended as an exhaustive account of either engineering or dance practice and their potential syntheses. Neither do we intend to argue that the few ways of making this synthesis that have been noted independently by scholars and practitioners across the last half century are enough to change engineering practice to the degree necessary for it impact cultures of mobility enough to enact significant environmental change. However we do believe, having noted that the limitation in engineering in facing is largely derived from its everyday modes of knowledge gathering, evaluation, and design, that it is exactly the kinds of small-scale epistemological and methodological tools in evident in dance that could change its efficacy.

It has been argued that engineering needs to change its assumptions about the kinds of effects it can have on urban environments and the tools it has to bring about change if cities are to be viable human habitats for the future. In order to do so, it will require transformative epistemological and methodological approaches that allow new options to emerge from design processes, rather than a futures-oriented approach that identifies hypothetical outcomes with no new ways to reach them. We conclude that there is clear need for further research to reveal further of these tools within dance, test out their incorporation into engineering projects through practice-based research, and observe whether new solutions are indeed made possible as has been predicted will be the case if engineering expanded its horizons.

Acarón, T., 2016. Shape-in(g) Space: Body, Boundaries, and Violence. Space Cult. 19, 139-149. https://doi.org/10.1177/1206331215623208

Agrawal, Roma. Built: The Hidden Stories Behind Our Structures. Bloomsbury Publishing, 2018.

Blockley, D., Godfrey, P., 2017. Doing It Differently - Second edition, 2nd edition. ed. ICE Publishing, London.

Bridge, G., Watson, S., 2011. Reflections on Mobilities, in: Bridge, G., Watson, S. (Eds.), The New Blackwell Companion to the City. Wiley-Blackwell, pp. 155-168. https://doi.org/10.1002/9781444395105.ch14

Bucciarelli, L.L., 2002. Between thought and object in engineering design. Des. Stud., Philosophy of Design 23, 219-231. https://doi.org/10.1016/S0142-694X(01)00035-7

Docherty, I., Giuliano, G., Houston, D., 2008. Connected Cities, in: Knowles, R., Shaw, J., Docherty, I. (Eds.), Transport Geographies : Mobilities, Flows and Spaces. Blackwell, Oxford \& Malden, MA, pp. 83-101.

Douglas, E.P., Koro-Ljungberg, M., Borrego, M., 2010. Challenges and promises of overcoming epistemological and methodological partiality: Advancing engineering education through acceptance of diverse ways of knowing. Eur. J. Eng. Educ. 35, 247-257. https://doi.org/10.1080/03043791003703177

Grimson, W., Murphy, M., Christensen, S.H., Erno-Kjolhede, E., 2008. Philosophy matters in engineering studies, in: 2008 38th Annual Frontiers in Education Conference. Presented at the 2008 38th Annual Frontiers in Education Conference, p. S4H-19-S4H-24. https://doi.org/10.1109/FIE.2008.4720424 
Hanoch-Roe, G., 2007. Scoring the Path: Linear Sequences in Music and Space, in: Muecke, M.W., Zach, M.S. (Eds.), Essays on the Intersection of Music and Architecture. Culicidae Architectural Press, Ames, IA, pp. 77-143.

Hillier, B., 2004. Space is the Machine. Space Syntax Ltd, London.

Hirsch, A.B., 2014. City Choreographer: Lawrence Halprin in Urban Renewal America. University of Minnesota Press.

Holling, C.S., 1973. Resilience and Stability of Ecological Systems. Annu. Rev. Ecol. Syst. 4, 1-23.

Hunter, V., 2017. Perecquian Perspectives: Dialogues with Site-Dance (Or, "On being here and there"). Lit. Geogr. 3, 27-49.

Jacobs, J., 1987. The uses of sidewalks: Contact, in: Glazer, N., Lilla, M. (Eds.), The Public Face of Architecture: Civic Culture and Public Spaces. pp. 95-112.

Jensen, O.B., 2006. "Facework", Flow and the City: Simmel, Goffman, and Mobility in the Contemporary City. Mobilities 1, 143-165. https://doi.org/10.1080/17450100600726506

Kanter, R.M., 2015. Move: Putting America's Infrastructure Back in the Lead, 1 edition. ed. W. W. Norton \& Company, New York.

Kato, S., Glynn, R., 2016. Fabricating Performance: A dance of circular feedback processes in constructing spatial notion, in: IN-PLAY. Presented at the Architecture InPlay, Instituto Universitário de Lisboa, Lisbon, pp. 6-13.

Kirsh, D., 2011. How Marking in Dance Constitutes Thinking with the Body. Extern. Mind 183-214.

Kroes, P., Franssen, M., Bucciarelli, L., 2009. Rationality in Design, in: Meijers, A. (Ed.), Philosophy of Technology and Engineering Sciences, Handbook of the Philosophy of Science. NorthHolland, Amsterdam, pp. 565-600. https://doi.org/10.1016/B978-0-444-51667-1.50025-2

Lam, T.F., 2017. Hackney: a cycling borough for whom? Appl. Mobilities 1-18. https://doi.org/10.1080/23800127.2017.1305151

Lanzendorf, M., Busch-Geertsema, A., 2015. Mode Decisions and Context Change? What About the Attitudes? A Conceptual Framework, in: Sustainable Urban Transport, Transport and Sustainability. Emerald Group Publishing Limited, pp. 23-42. https://doi.org/10.1108/S2044994120150000007012

Low, N., O'Connor, K., 2013. The Dilemma of Mobility, in: Low, N. (Ed.), Transforming Urban Transport. The Ethics, Politics and Practices of Sustainable Mobility, Earthscan. Routledge, London, pp. 3-25.

McCormack, D.P., 2008. Geographies for Moving Bodies: Thinking, Dancing, Spaces. Geogr. Compass 2, 1822-1836. https://doi.org/10.1111/j.1749-8198.2008.00159.x

Merriman, P., 2011. Roads: Lawrence Halprin, modern dance and the American freeway landscape, in: Geographies of Mobilities: Practices, Spaces, Subjects. Ashgate, Farnham, UK, pp. 99-118.

Prytherch, D., 2015. Rules of the Road: Choreographing Mobility in the Everyday Intersection, in: Julie Cidell, David Prytherch (Eds.), Transport, Mobility, and the Production of Urban Space, Human Geography. Routledge, Abingdon, pp. 45-63. https://doi.org/10.4324/9781315709680

Ramírez, F., Seco, A., 2012. Civil Engineering at the Crossroads in the Twenty-First Century. Sci. Eng. Ethics 18, 681-687. https://doi.org/10.1007/s11948-011-9258-2

Schiller, G., Rubidge, S. (Eds.), 2014. Choreographic Dwellings: Practising Place. Palgrave Macmillan, Basingstoke.

Seamon, D., 2007. A Lived Hermetic of People and Place: Phenomenology and Space Syntax, in: Proceedings. Presented at the 6th International Space Syntax Symposium, Istanbul Technical University, Istanbul.

Seamon, D., 1979. A geography of the lifeworld: movement, rest, and encounter, Croom Helm series in geography and environment. Croom Helm.

Spinks, N., Silburn, N., Birchall, D., 2006. Educating engineers for the 21st century: The industry view. Lond. R. Acad. Eng.

Symes, Colin. 'Entr' acte: Mobile Choreography and Sydney Rail Commuters'. Mobilities 8, no. 4 (1 November 2013): 542-59. https://doi.org/10.1080/17450101.2012.724840.

Transport for London, 2017. Healthy Streets for London: Prioritising walking, cycling and public transport to create a healthy city. TfL, London. 
(2) 